Kuzdzal-Savote S. et Kuzdzal W. Ann. Biol. anim. Bioch. Biophys., 1961,1 (1), 47.

Kuzdzal-Savore S. et Kuzdzal W. XVI Cong. Intern. Lait, 1962, Copenhague.

LAXA O. Ann. Fals., 1931, 24, 87.

De la Mare P. B. D. et Shorland F. B. Nature, 1944, 153, 380.

Salmon-Legagneur E. Ann. Zoot., 1960, 8 (4), 349 (356).

SмIтн L. M. J. Dairy Sci., 1961, 44, 607.

\title{
VALEUR ALIMENTAIRE DU LAIT (1)
}

\author{
par le Docteur J. TREMOLIERES, \\ Président du Comité de Nutrition \\ de la Délégation Générale à la Recherche
}

La valeur alimentaire est souvent assimilée à la valeur nutritionnelle et l'on s'étonne alors qu'une denrée qui a tant de titres de noblesse soit moins bien acceptée et moins consommée qu'on ne le voudrait.

\section{Valeur nutritionnelle}

TABLEAU I

TENEUR EN PROTÉINES DU LAIT DE VACHE (G POUR 100 G)

\begin{tabular}{|c|c|c|c|c|}
\hline & $\begin{array}{l}\text { Protéines } \\
\mathrm{N} \times 6,25\end{array}$ & Lipides & Glucides & Calories \\
\hline \multirow[t]{2}{*}{ Frais entier } & $3,3(2)$ & 3,7 & 4,8 & 66 \\
\hline & $3,3(3)$ & 4,0 & 5 & 71 \\
\hline \multirow[t]{2}{*}{ Frais écrémé $\ldots \ldots \ldots \ldots$} & $3,4(2)$ & 0,2 & 5,1 & 35 \\
\hline & $3,7(3)$ & 0,2 & 5 & 37 \\
\hline \multirow[t]{2}{*}{ Condensé entier sucré } & $8,0(2)$ & 12,0 & 56,0 & 354 \\
\hline & $8,8(3)$ & 8,3 & 54,1 & 340 \\
\hline \multirow{2}{*}{$\begin{aligned} \text { Condensé entier } & \text { non } \\
\text { sucré (évaporé) } & \ldots \ldots\end{aligned}$} & $7,6(2)$ & 8,4 & 12,3 & 155 \\
\hline & $6,7(3)$ & 8,2 & 10,1 & 133 \\
\hline Condensé écrémé sucré .. & $9,7(2)$ & 0,3 & 60 & 267 \\
\hline En poudre écrémé $\ldots \ldots$ & $34,0(2)$ & 0,3 & 49,2 & 600 \\
\hline \multirow[t]{2}{*}{ En poudre entier $\ldots$} & $26,4(2)$ & 29,7 & 38,8 & 530 \\
\hline & $28,7(3)$ & 26,9 & 36,5 & 500 \\
\hline
\end{tabular}

(1) Cahiers des Ingénieurs agronomes, 1963, n० 175, 11.

(2) McCance et Widdowson. The chemical composition of foods, M.R.S., 1946.

(3) Bridges' foods and beverage analysis. M. Mattice, Lea et Febiger, 1951. 
Pour qu'une denrée soit mangée, il ne suffit pas qu'elle soit "rationnelle". il faut aussi qu'elle soit "désirée " et qu'elle soit bien considérée.

On a assez dit que le lait est un aliment complet et équilibré, pour qu'il soit inutile d'y insister. La valeur biologique de ses protéines figure parmi les plus élevées. Leur richesse en lysine en fait le complément naturel des protéines des céréales.

\section{TABLEAU II}

SUPPLÉMENTATION ENTRE LES PROTÉINES DU PAIN, DU FROMAGE, DES POMMES DE TERRE ET DU LAIT,

ET INFLUENCE DU LAPS DE TEMPS INTERMEDIAIRE SUR GES APPORTS

\begin{tabular}{|c|c|}
\hline Origine des protéines & $\begin{array}{l}\text { Valeur biologique des pro- } \\
\text { téines pour les rats }\end{array}$ \\
\hline Pain blane $\ldots \ldots \ldots \ldots \ldots \ldots \ldots \ldots \ldots$ & 52 \\
\hline Fromage Cheddar $\ldots \ldots \ldots \ldots \ldots \ldots \ldots \ldots \ldots$ & 76 \\
\hline 1 partie fromage $+3,4$ parties de pain $(+)$ : & \\
\hline - simultanément $\ldots \ldots \ldots \ldots \ldots \ldots \ldots \ldots$ & 76 \\
\hline 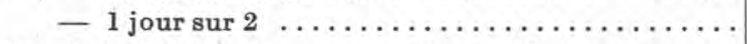 & 67 \\
\hline $\begin{array}{l}\text { La valeur biologique supposée, si l'apport supplé- } \\
\text { mentaire n'existait pas, serait de } \ldots \ldots \ldots \ldots \ldots \ldots\end{array}$ & 64 \\
\hline Pommes de terre $\ldots \ldots \ldots \ldots \ldots \ldots \ldots \ldots \ldots$ & 71 \\
\hline 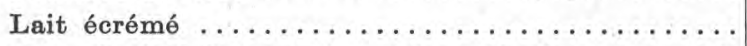 & 89 \\
\hline $\begin{array}{l}1 \text { partie de lait écrémé }+1,3 \text { partie de pommes de } \\
\text { terre : }(+)\end{array}$ & \\
\hline - simultanément $\ldots \ldots \ldots \ldots \ldots \ldots \ldots \ldots$ & 86 \\
\hline-1 joursur $2 \ldots \ldots \ldots \ldots \ldots \ldots \ldots \ldots$ & 81 \\
\hline $\begin{array}{l}\text { La valeur biologique supposée, si l'apport supplé- } \\
\text { mentaire n'existait pas, serait de } \ldots \ldots \ldots \ldots \ldots\end{array}$ & 80 \\
\hline
\end{tabular}

(+) Fourniture de la même quantité d'azote par ces deux produits.

Le lait est la source majeure de vitamine B2, de calcium, de vitamine A et de vitamine D. Il n'est déficitaire qu'en fer et en vitamine $\mathrm{C}$, et dans certaines conditions en acides gras essentiels. La teneur en graisse du lait entier de vache est trop élevée (environ 50 p. 100 de calories grasses) alors qu'il est conseillé à l'homme d'avoir une ration ne comportant pas plus de 35 p. 100 de calories grasses.

Et cependant, alors que 95 p. 100 des parents et des enfants savent cette valeur nutritionnelle remarquable, 65 p. 100 des garçons et 55 p. 100 des filles seulement acceptent de boire régulièrement le bol de lait qu'on leur offre, d'après une enquête faite par l'Institut National d'Hygiène. Il y a done là un problème d'acceptabilité. 


\section{Valeur psychosensorielle}

- Il résulte d'une enquête faite aux U.S.A. en 1943, sous la direction de M. Mead que même l'Américain boit le lait "par raison", parce que c'est "bon pour la santé ", mais que, même dans un type alimentaire assez "délipidinisé ", il n'y a pas, pour le lait, d'appétence spéciale comparable à celle du beefsteack.

- Une des enquêtes faites par l'Institut National d'Hygiène à Marseille, a montré que les qualités organoleptiques du lait jouent un grand rôle dans son acceptabilité. Le désir de consommer le lait n'apparaît que pour un lait qui soit bon au goût, le "bon goût " devant être mieux défini.

Le tableau suivant donne les coefficients par lesquels il faut multiplier le prix d'un litre de lait pour obtenir le prix que devraient avoir les autres denrées pour coûter le même prix au gramme de protéines :

\section{TABLEAU III}

Le prix du litre de lait devrait être le prix du kilo des denrées suivantes, divisé par les coefficients ci-dessous, pour que le gramme de protéine soit payé le même prix. (Ex. : si le beefsteack coûte 10 franes le kilo, le lait devrait coûter 10 franes divisé par $5=2 \mathrm{~F}$ le litre, pour que le gramme de protéine soit payé le même prix.)

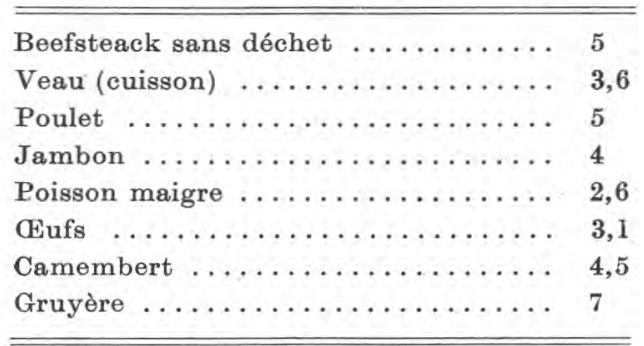

- Ainsi, bien que le lait offre le gramme de protéines animales le moins cher, sa consommation en France, reste la plus basse parmi celle des pays industriels.

Le tableau $\mathrm{V}$ présente les consommations par individu moyen et par jour de produits laitiers, d'après les enquêtes de l'Institut National d'Hygiène.

\section{Facteurs de l'acceptabilité}

Nous ne pouvons donner ici qu'un aperçu partiellement hypothétique, renvoyant pour les détails aux travaux cités en bibliographie. Autant une importante masse de travaux a été consacrée 
TABLEAU IV

CONSOMMATION EN KG PAR TETE ET PAR AN, EN FRANGE ET DANS QUELQUES PAYS ÉTRANGERS (1)

\begin{tabular}{|c|c|c|c|c|c|c|c|}
\hline 1 & $\begin{array}{c}\text { Prance } \\
\text { (5) }\end{array}$ & $\begin{array}{l}\text { Allemagne } \\
\text { Fédérale }\end{array}$ & $\begin{array}{l}\text { Belgique } \\
\text { Luxemb. }\end{array}$ & Pays-Bas & Italie & $\begin{array}{c}\text { Royaume- } \\
\text { Uni }\end{array}$ & $\begin{array}{l}\text { Etats- } \\
\text { Unts }\end{array}$ \\
\hline Viandes $\ldots . . . \ldots \ldots$ & 68,7 & 48,1 & 53,0 & 38,4 & 20,4 & $67,6 \quad(4)$ & 81,5 \\
\hline dont bœuf ........ & 21,2 & 14,6 & $\begin{array}{r}20,6 \\
(2)\end{array}$ & 15,3 & $\begin{array}{r}10,0 \\
(2)\end{array}$ & $\begin{array}{r}22,3 \\
(2)\end{array}$ & 29,6 \\
\hline Pore et charcuterie .. & 18,5 & 26,2 & 23,5 & 14,9 & 4,0 & 7,9 & 27,3 \\
\hline Volailles ........... & 6,9 & 1,8 & $\begin{array}{r}2,5 \\
(3)\end{array}$ & 0,6 & 2,0 & 3,3 & 12,6 \\
\hline CEufs $(1 œ u f=55 \mathrm{~g}) \ldots$ & 10,3 & 10,4 & 14,4 & 8,2 & 7,8 & 12,4 & 21,2 \\
\hline Poissons ............ & 10,2 & 7,1 & 11,6 & 8,0 & 6,9 & 9,8 & 4,8 \\
\hline $\begin{array}{l}\text { Lait frais } \\
\qquad(11=1,033 \mathrm{~g}) \ldots \ldots\end{array}$ & 89,1 & 124,7 & 91,1 & 197,2 & 55,4 & & 162,9 \\
\hline Fromage $\ldots \ldots \ldots$ & 7,1 & 6,1 & 5,5 & 6,3 & 6,5 & 4,2 & 5,7 \\
\hline Corps gras ......... & 17,0 & 25,2 & 22,9 & 27,6 & 13,8 & 24,5 & 22,0 \\
\hline dont beurre ....... & 5,8 & 5,8 & 9,2 & 2,7 & 1,2 & 6,9 & 4,0 \\
\hline huiles et autres & 11,2 & 19,4 & 12,8 & 24,9 & 12,6 & 17,6 & 18,0 \\
\hline
\end{tabular}

(1) Moyenne quadriennale 1954-55-56-57 (en kgs) (OECE).

(2) Bouf et veau.

(3) Volailles, lapins et gibiers.

(4) Chiffires incertains.

(5) Les chiffres indigués ici pour la France sont inférieurs de 20 à $30 \%$ aux sondages de consommation des enquêtes de l'Institut National d'Hygiène.

à la valeur nutritionnelle, autant on n'a guère que des indications sur les facteurs de l'acceptabilité.

a) Le lait fait sécréter une salive muqueuse, épaisse et peu rafraîchissante. Cette propriété dépend de la température du lait, de son acidité. Elle est peut-être en rapport avec la forme sous laquelle se trouve le calcium, et avec les caractéristiques de l'émulsion grasse. Toujours est-il qu'elle diffère suivant les laits et les traitements subis et qu'il serait utile d'en savoir davantage sur le type de traitement à appliquer au lait pour que celui-ci donne la sensation la plus rafraîchissante. Il est probable que c'est la pasteurisation dite A.T.S.T. ( $72^{\circ} \mathrm{C}$ pendant 15 secondes) avec homogénéisation et consommation de lait froid, sans que ce dernier ait été à aucun moment porté à l'ébullition.

b) Le lactose du lait est absorbé par le grêle, après dédoublement par la lactase de l'épithélium intestinal. Or, il est probable que la lactase est une enzyme adaptative dont le taux se réduit beaucoup dans la muqueuse duodénale quand on cesse de boire 


\section{TABLEAU V}

CONSOMMATION EN GRAMMES PAR INDIVIDU MOXEN ET PAR JOUR DE PRODUITS LAITIERS (ENQUETE DE L'INSTITUT NATIONAL D'HYGIẺNE)

\begin{tabular}{|c|c|c|c|c|c|c|}
\hline & $\begin{array}{c}\text { Familles } \\
\text { urbaines } \\
1949-1951 \\
(1)\end{array}$ & $\begin{array}{c}\text { Familles } \\
\text { rurales } \\
1949-1951 \\
(2)\end{array}$ & $\begin{array}{c}\text { Familles } \\
\text { urbaines } \\
1952-1956 \\
(3)\end{array}$ & $\begin{array}{c}\text { Familles } \\
\text { rurales } \\
1952-1957 \\
(4)\end{array}$ & $\begin{array}{c}\text { Familles } \\
\text { urbaines } \\
1958-1959 \\
(5)\end{array}$ & $\begin{array}{c}\text { Familles } \\
\text { rurales hiver } \\
1959-1960 \\
\text { (6) }\end{array}$ \\
\hline Lait entier $\ldots \ldots \ldots$ & 314 & 325 & 271 & 301 & 301 & 359 \\
\hline Lait écrémé $\ldots \ldots \ldots$ & - & 21 & - & - & - & - \\
\hline Lait conservé ...... & - & - & - & - & - & - \\
\hline Fromage $\ldots \ldots \ldots \ldots$ & 26 & 16,8 & 23 & $\begin{array}{c}12,5 \\
(\times 6=65 \\
\text { en lait })\end{array}$ & 46,4 & 19,4 \\
\hline Total ........ & 340 & 362,8 & 294 & 313,5 & 347,4 & 378,4 \\
\hline Crème fraîche $\ldots . .$. & - & - & - & $(*)$ & 0,3 & 11 \\
\hline Beurre $\ldots \ldots \ldots \ldots$ & 23 & 30,5 & 24 & 40 & 24,7 & 48,4 \\
\hline Huile, lard, saindoux . & - & - & 33 & 28,5 & 23 & 22 \\
\hline Autres graisses ...... & 31 & 16 & - & & & \\
\hline Margarine....... & & & 9 & 4 & 4,1 & 6 \\
\hline Total ......... & 54 & 46,5 & 66 & 78,5 & 52,1 & 87,4 \\
\hline \multicolumn{7}{|c|}{$\begin{array}{l}\text { (1) Paris 1949, Nancy 1949-1950, Marseille 1950, Saint-Etienne 1950, Strasbourg 1951, Paris } 1951 . \\
\text { (2) Bretagne, Mayenne, Normandie, Anjou, Vendée, Pays Nantais, Monts du Lyonnais, Auvergne, } \\
\text { Bourgogne, Lorraine, Haute-Marne, Limousin, Gard, Adour, Gascogne. }\end{array}$} \\
\hline \multicolumn{7}{|c|}{$\begin{array}{l}\text { (3) Marseille 1952-1953, Nancy et Meurthe-et-Moselle 1952-1953, 1954-1955, Vosges et Moselle } 1956 . \\
\text { (4) Meurthe et Moselle 1952-53-54-55, Vendée 1955, Sarthe 1957. }\end{array}$} \\
\hline \multicolumn{7}{|c|}{ (5) Lyon, Marseille, Saint-Etienne 1958-1959; Nantes 1959.} \\
\hline \multicolumn{7}{|c|}{ (6) Loire-Atlantique 1959 ; Basse-Normandie 1960.} \\
\hline (*) Consommation de b & eurre en Meu & rthe-et-Mosel & $\mathrm{e}: 30,5 ; \mathrm{Sa}$ & ae: 36 ; Ven & : 66. & \\
\hline
\end{tabular}

du lait. Ainsi les tentatives pour reprendre des quantités notables de lait sont-elles volontiers accompagnées de diarrhée ou d'impression de ballonnement. Donc l'habitude perdue est difficile à reprendre.

c) Le lait semble avoir un pouvoir excito-moteur assez faible sur les sécrétions gastrique et pancréatique, d'après les expériences de Pavlov. La quantité totale de pepsine sécrétée, après ingestion de la même quantité de protéines de viande, de pain ou de lait, est 2 fois plus grande pour la viande et 5 fois pour le pain.

d) Par contre, le beurre semble, jusqu'à nouvel ordre, la graisse demandant le travail digestif le plus faible, ayant l'absorption digestive la plus rapide et dont les effets sur la contraction de la vésicule biliaire sont les plus doux. 


\section{Prestige social}

Actuellement, en France, d'après les enquêtes de l'Institut National d'Hygiène, la consommation du lait n'est élevée que dans les familles nombreuses et, dans certaines régions rurales (Jura, Massif Central) où on a gardé l'habitude du café au lait le soir. Elle disparaît de façon inquiétante des petits déjeûners de l'écolier. Et cependant la journée continue, avec son repas de midi rapide, gagnerait probablement à de simples ingestions liquides nourrissantes, telles que des boissons lactées acidifiées ou non, pourraient le constituer.

\section{en pratique}

- La valeur alimentaire du lait, indiscutable sur le plan nutritionnel, est limité par son acceptabilité. Cette acceptabilité dépend de facteurs complexes que nous avons énumérés.

- Le marché du lait-boisson n'existe guère en France, où en raison d'un soi-disant danger bactériologique que l'on pourrait parfaitement éviter par des méthodes de production et de pasteurisation adéquate, on écarte un intérêt nutritionnel certain et une solution partielle à des problèmes économiques aigus. La réalisation d'un bon lait boisson demanderait à la base un lait possédant d'excellentes qualités organoleptiques, assaini sur le plan bactériologique par une pasteurisation à basse température pendant un temps bref, une homogénéisation, une consommation froide. Une gamme des boissons lactées acidifiées, demi-écrémées, serait également à réaliser.

- Ce lait-boisson constituerait l'aliment de choix pour les collations de la journée continue ou des compléments nutritionnels équilibrant dans l'alimentation de l'écolier et de l'étudiant.

- L'habitude de boire du lait une fois perdue, est difficile à reprendre, probablement par réduction du pouvoir d'absorber le lactose. Une réelle politique du lait-boisson serait à mettre sur pied dans l'intérêt de la Santé Publique et de l'économie.

\section{BIBLIOGRAPHIE}

[1] Séminaire de Bastad, publié par la Fédération Internationale de Lairie, 1961.

[2] J. Trémolı̀̀res. / Matière azotée du lait. Intérêt pour la nutrition humaine. Journée d'Etude et d'Information de l'Association Laitière Française et du Centre Interprofessionnel Laitier du Bassin Lyonnais, Lyon, 27 octobre 1961.

[3] J. Trémolik̀res. La matière grasse du lait et la nutrition. Journée d'Etude sur la qualité du beurre. Rennes, 25 mai 1961.

[4] XVIe Congrès International de Laiterie. Copenhague, 1962. 


\title{
Summary
}

It would be very advantageous that the useful microorganismes, which are destroyed by antibiotics, should be provided to the human body by food. Cheeses might be this food, because their microbial flora is important and various.

\section{AURONS-NOUS DU BIO-FROMAGE? (1)}

\author{
par \\ ANDRÉ MOLDAVAN
}

Lors du récent congrès, à Montréal, de l'Association des Médecins de langue française de l'Amérique, le $D^{r}$ René Dubos, de l'Institut Rockefeller, dans sa conférence sur les antibiotiques faisait trois remarques d'un intérêt spécial pour l'industrie laitière.

1. - Certains germes pathogènes, en présence d'antibiotiques, se transforment en souches nouvelles résistantes à ces antibiotiques. Ces persisteurs perdent temporairement leur virulence mais restent une source possible d'infections ultérieures.

2. - Les antibiotiques détruisent spécifiquement certains germes pathogènes dans le corps mais en même temps, attaquent un grand nombre de microbes utiles à la santé, particulièrement ceux des intestins. Il en résulte une diminution de la résistance naturelle du corps suivie parfois d'infections gastro-intestinales secondaires.

3. - Le $\mathrm{D}^{\mathrm{r}}$ Dubos a exprimé l'espoir que la microbiologie s'efforcera d'identifier et de cultiver ces microbes utiles et bienfaisants afin de pouvoir les ensemencer à nouveau dans les intestins du patient après le traitement antibiotique.

\section{Microbiologie intestinale}

Nos connaissances de la microbiologie pathologique des intestins sont beaucoup plus vastes que celles de la microbiologie naturelle des intestins en bonne santé.

L'estomac et le duodénum de l'intestin grêle ont une population microbienne relativement faible, mais celle-ci augmente dans le jéjunum et dans l'iléum. Le colon ou gros intestin contient une

(1) Le Québec laitier, janvier 1963. 